03;01.1;09.2

\title{
Оптимизация параметров газогенераторной и лидарной систем для измерения потоков воздуха при неразрушающем контроле качества охлаждающих микроканалов на малых расстояниях
}

\author{
(C) А.А. Ким ${ }^{1,2}$, К.В. Елисеев ${ }^{1}$, В.В. Акапьев ${ }^{1}$, В.Л. Акапьев ${ }^{3}$, С.Е. Савотченко ${ }^{4}$, А.С. Горлов ${ }^{4}$ \\ ${ }^{1}$ Балтийский государственный технический университет „ВОЕНМЕХ“ им. Д.Ф. Устинова, Санкт-Петербург, Россия \\ ${ }^{2}$ АО „Лазерные системы“, Санкт-Петербург, Стрельна, Россия \\ ${ }^{3}$ Белгородский юридический институт МВД России им. И.Д. Путилина, Белгород, Россия \\ ${ }^{4}$ Белгородский государственный технологический университет им. В.Г. Шухова, Белгород, Россия \\ ฯ E-mail: savotchenkose@mail.ru
}

Поступило в Редакцию 12 июля 2019г.

В окончательной редакции 10 фревраля 2020г.

Принято к публикации 10 февраля 2020 г.

\begin{abstract}
Описывается метод неразрушающего контроля качества состояния охлаждающих микроканалов высоконагруженных турбинных лопаток путем измерения скорости истекающего аэрозольсодержащего воздушного потока с помощью когерентного доплеровского непрерывного лидара. Разработана математическая модель, с помощью которой определены оптимальные значения параметров газогенераторной и лидарной систем. Исследовано влияние диаметра частиц аэрозоля на мощность обратного рассеяния.
\end{abstract}

Ключевые слова: доплеровский лидар, неразрушающий контроль качества, обратное рассеяние, аэрозоль, сечение рассеяния.

DOI: 10.21883/PJTF.2020.09.49367.17977

При производстве и обслуживании высоконагруженных турбинных лопаток существенной проблемой является контроль состояния охлаждающих микроканалов. Лопатки высоконагруженных турбин во время работы испытывают огромные термические и механические воздействия. Температура протекающего газа может достигать $1500-1700^{\circ} \mathrm{C}$ и более, при этом предел прочности лопаток, изготавливаемых из жаропрочных сталей, часто оказывается ниже и не превосходит температур порядка $1300-1350^{\circ} \mathrm{C}$ [1]. Сегодня эта проблема чаще всего решается путем создания особых охлаждающих микроканалов, соединяющих внутренние полости лопатки с ее внешней поверхностью. В этом случае происходит достаточно эффективное конвективно-пленочное охлаждение при истекании охлаждающего газа из микроканалов [1]. Поскольку турбинные лопатки работают в критических условиях, незначительный дефект микроканала и неравномерность охлаждения могут привести к локальному перегреву и быстрому разрушению лопатки и турбинного агрегата. Этим фактом обусловливаются высокие требования к качеству изготовления самих лопаток и микроканалов охлаждения. Типичный диаметр микроканала составляет 200-300 $\mu \mathrm{m}$, что вызывает существенные затруднения при контроле их состояния и качества изготовления.

В настоящее время для решения подобных задач могут быть использованы различные методы, такие как применение сканеров белого света, оптических компараторов, компьютерная томография [2,3], а также контактные зондовые методы. Недостатки оптических методов, основанных на анализе изображений поверхности лопат- ки, заключаются в невозможности измерений внутренних размеров и пропускания микроканалов. Компьютерная томография позволяет проанализировать структуру каналов, однако обладает невысокой точностью и не исключает возможности пропуска или нераспознавания скрытого дефекта. На данный момент только зондовые методы обеспечивают достаточно высокую вероятность обнаружения дефектного микроканала, однако они обладают очень низкой производительностью и требуют дорогостоящих расходных материалов. Таким образом, разработка методов и средств неразрушающего контроля состояния микроканалов турбинных лопаток является актуальной научно-технической задачей [4-7].

Перспективным методом неразрушающего контроля микроканалов можно считать применение гетеродинного доплеровского лидара [5] для измерения скорости истекания микропотоков воздуха. Устройства подобного типа широко применяются в метеорологии для зондирования атмосферы и построения различных пространственных профилей скорости и направления ветра [8]. Сигналом для доплеровских лидаров является оптическое излучение, обратно рассеянное от каждой точки атмосферной трассы, содержащей мелкодисперсные частицы аэрозоля (водяного пара). Идея контроля качества охлаждающих микроканалов заключается в том, что при продувании их газовой аэрозольсодержащей смесью с известным давлением возможна оценка эффективной площади каждого микроканала и его пропускания путем анализа скорости истекающего газового потока.

Основной целью настоящей работы является оптимизация физических параметров на основе моделиро- 
вания оптической и газогенераторной систем, предназначенных для измерения объектов, находящихся в непосредственной близости от оптического измерителя. Существенная малость расстояния от объекта до измерителя обусловливает появление новых конструктивных требований к системе, а также к ее физическим параметрам. В результате возникает необходимость изучения возникающих особенностей физических процессов, происходящих в системе, в частности распределения излучения, рассеянного частицами определенного размера при малых расстояниях между регистратором и измеряемым объектом. Задача оптимизации состоит в нахождении таких значений диаметра частиц аэрозоля, мощности обратного рассеяния и других параметров, при которых будут достигаться требуемые характеристики технических компонентов лидара.

Метод лидарного измерения скорости газа основан на регистрации эффекта Доплера при отражении (обратном рассеянии) узкополосного оптического излучения от аэрозольных частиц с ненулевой проекцией скорости на ось зондирования. Строго говоря, измеряется не сама скорость газа, а лишь проекция ее вектора на направление распространения оптического излучения. При отражении электромагнитной волны с частотой $v$ от подвижного объекта (газа) со скоростью $V$, согласно закону Доплера, происходит смещение частоты излучения $v^{\prime}$ :

$$
v^{\prime}=v\left(1 \pm 2 V_{z} / c\right),
$$

где $V_{z}$ - проекция скорости частиц газа на направление распространения излучения, $c$ - скорость света. Доплеровский когерентный лидар определяет радиальную проекцию скорости зондируемого объекта путем вычисления величины доплеровского смещения частоты при когерентном (гетеродинном) сложении опорного сигнала лазера и сигнала, отраженного от объекта зондирования. Как известно, при сложении двух квазикогерентных сигналов (различающихся по частоте) в области их интерференции возникает амплитудное биение, частота которого равна разности их частот, в данном случае величине доплеровского сдвига частоты. Фурье-анализ электрического сигнала с фотоприемника лидара позволяет определить доплеровскую спектральную компоненту и вычислить скорость истекания газа. В случае использования лидара для контроля качества изготовления охлаждающих микроканалов требуется измерение скорости истекания газа из каждого канала контролируемого изделия. При известном давлении газовоаэрозольной смеси и номинальном диаметре охлаждающих каналов нетрудно оценить скорость истекания газа. Любые отклонения измеренной скорости от этих значений в бо́льшую или меньшую сторону могут быть интерпретированы как дефект.

Специфика рассматриваемой лидарной системы по сравнению с метеорологическими лидарами заключается в том, что точка измерения находится в непосредственной близости от приемопередающей оптики. Гео-

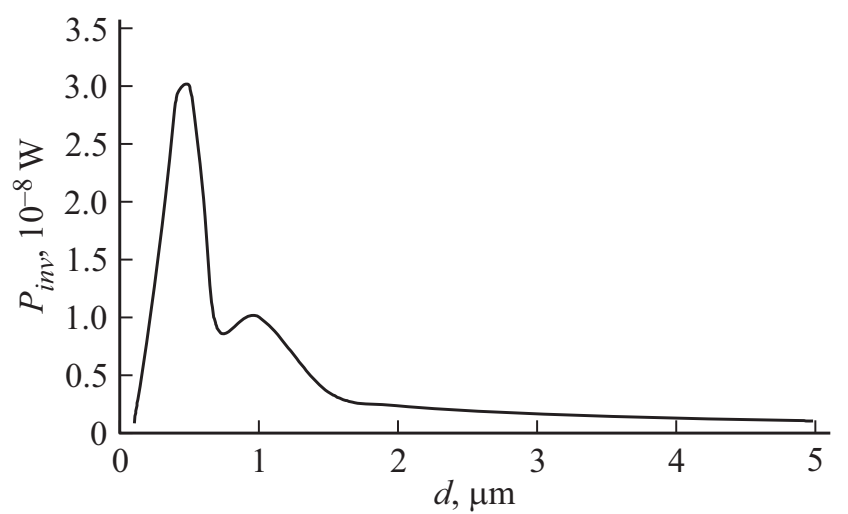

Рис. 1. Зависимость мощности обратного рассеяния от диаметра частиц аэрозоля.

метрические размеры области детектирования (гауссовой перетяжки в области фокуса телескопа) должны быть согласованы с параметрами струи истекающего газа на выходе из микроканала. Более того, в данном случае для эффективного детектирования обратно рассеянного излучения необходимо оптимизировать управляемые физические параметры - характеристики газово-аэрозольной смеси, в частности концентрацию аэрозольных частиц, их размер и давление газа, а также величину гауссовой перетяжки в точке фокусировки зондирующего излучения; критерий оптимальности максимизация мощности обратно рассеянного излучения и, следовательно, наибольший уровень сигнала. В качестве исходных данных для моделирования были выбраны значения длины волны излучения $\lambda \approx 1.5 \mu \mathrm{m}$ и мощности $1 \mathrm{~W}$. Такие значения характерны для доплеровских лидаров с гетеродинным приемом.

Далее в работе представлены результаты моделирования оптического блока для фокусировки лазерного излучения, приема рассеянного излучения и передачи его в лазерный блок. Основные оценки оптимальных параметров получены напрямую в рамках теории Ми средствами программного комплекса при использовании таких исходных значений параметров системы (длина волны $1.5 \mu \mathrm{m}$, диаметр сканируемого отверстия $200 \mu \mathrm{m}$, диапазон скоростей истекания аэрозоля из отверстия $5-100 \mathrm{~m} / \mathrm{s})$, при которых достигаются максимальное значение мощности обратного рассеяния и требуемый порог соотношения сигнал-шум.

Для оценки параметров основных блоков устройства было проведено моделирование и изучена зависимость мощности обратного рассеяния от управляемых параметров, таких как диаметр аэрозольной частицы $d$ и эффективные сечения обратного рассеяния $\sigma_{\pi}$ и поглощения $\sigma_{\alpha}$ :

$$
P_{i n v}=2 \beta_{\pi} P_{o u t} \pi \lambda \exp (-2 \alpha l)
$$

где $l-$ длина перетяжки, $P_{\text {out }}=1 \mathrm{~W}-$ мощность источника излучения, $\beta_{\pi}$ - коэффициент обратного рассеяния, $\alpha-$ коэффициент поглощения аэрозоля $[9,10]$. 


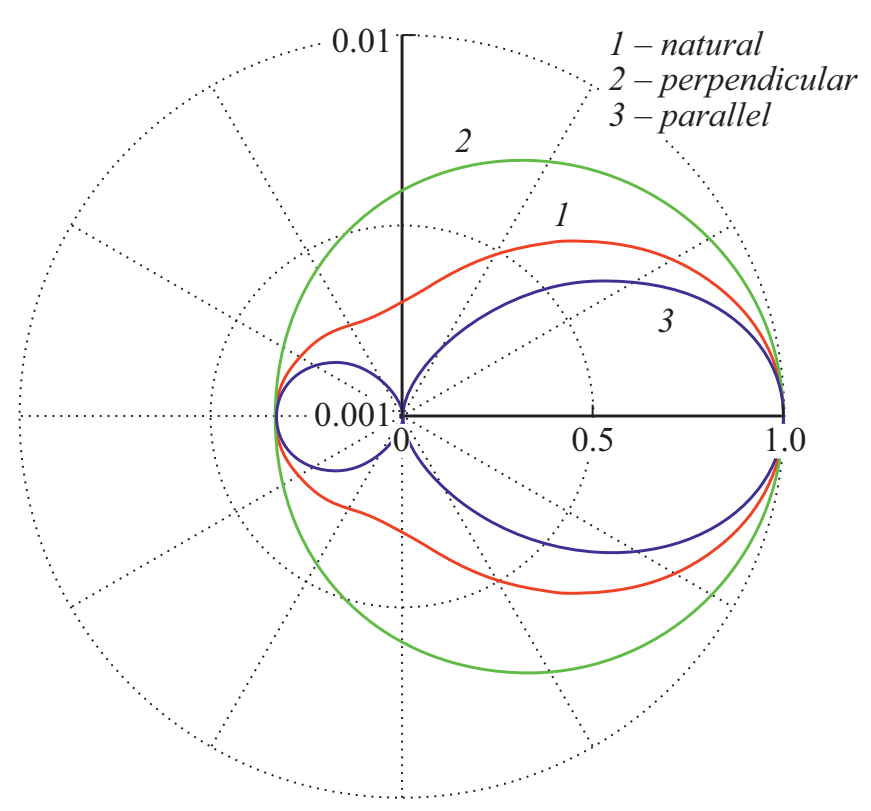

Рис. 2. Индикатриса рассеяния при диаметре частиц $0.5 \mu \mathrm{m}$.

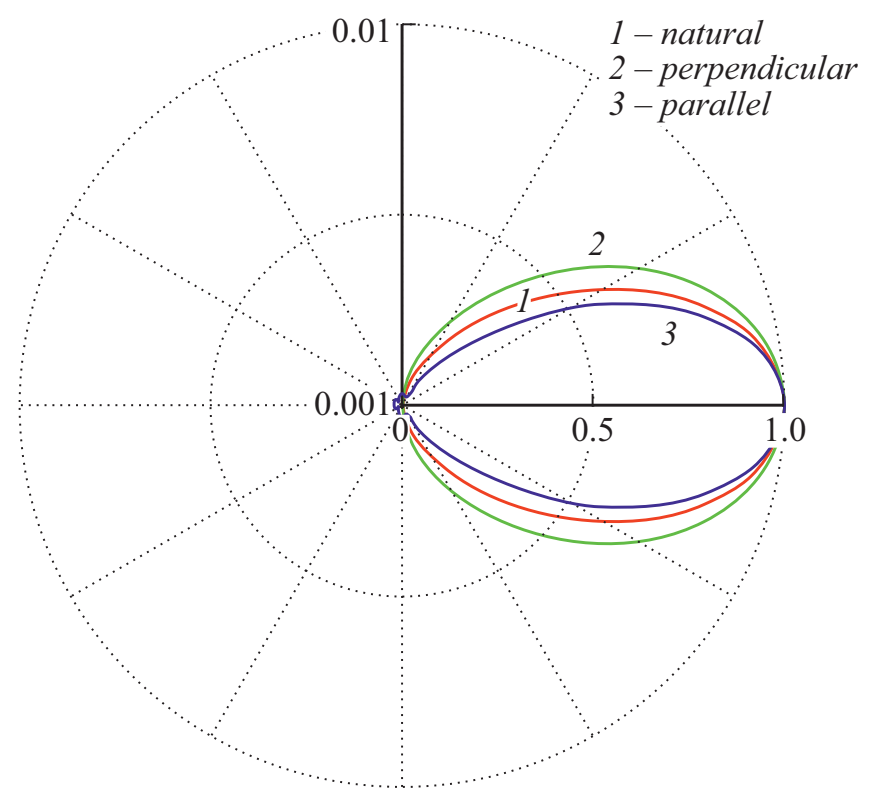

Рис. 3. Индикатриса рассеяния при диаметре частиц $1 \mu \mathrm{m}$.

Данные коэффициенты определяются соотношениями $\beta_{\pi}=N \sigma_{\pi}, \alpha=N \sigma_{\alpha}$, где $N-$ концентрация частиц аэрозоля. Длина перетяжки составляет две рэлеевские длины и определяется выражением [10]:

$$
l=4 f^{2} \lambda M^{2} / \pi D^{2},
$$

где $M^{2}$ - показатель качества пучка, $D$ - диаметр выходной оптики телескопа, $f$ - его фокусное расстояние. Тогда для исходных значений $M^{2} \geqslant 1, D=20 \mathrm{~mm}$ и $f=500 \mathrm{~mm}$ получается оценка длины перетяжки $l \approx 3 \mathrm{~mm}$.
В рамках математической модели, основанной на теории Ми $[11,12]$, для диаметра сферической рассеивающей частицы $1 \mu \mathrm{m}$, длины волны $\lambda=1.5 \mu \mathrm{m}$, реального коэффициента преломления воды 1.3 и его мнимой части (коэффициент поглощения) -0.74 , единичной концентрации частиц на один кубический микрометр были получены оценки значений эффективного сечения поглощения $\sigma_{\alpha} \approx 1.9 \mu \mathrm{m}^{2}$ и эффективного сечения обратного рассеяния $\sigma_{\pi} \approx 0.12 \mu \mathrm{m}^{2}$. Оценка показала, что, поскольку оптический путь в пределах перетяжки является малым, для полученных численных значений параметров $\alpha l \ll 1$, и поэтому $\exp (-2 \alpha l) \sim 1$, а значит, экспоненциальным множителем в формуле (2) можно пренебречь. В результате получается оценка мощности $P_{i n v} \approx 29 \mathrm{nW}$, на основании которой установлено, что для гетеродинного приема такой мощности обратного рассеяния достаточно использовать источник с мощностью $10 \mathrm{~mW}$.

Полученные значения сечений используются для исследования влияния диаметра частиц аэрозоля на мощность обратного рассеяния в рамках теории Ми. Результаты моделирования, приведенные на рис. 1, показали, что максимум мощности обратного рассеяния, величина которого составляет порядка $30 \mathrm{nW}$, соответствует диаметру частиц порядка $0.5 \mu \mathrm{m}$. Видно, что с увеличением диаметра частицы мощность обратного рассеяния падает. Следует отметить, что зависимость мощности обратного рассеяния от диаметра частиц аэрозоля обычно носит немонотонный характер. В частности, в [8] показано, что зависимость фактора эффективности обратного рассеяния от размера частиц водного аэрозоля убывает осциллирующим образом, причем значения максимумов амплитуд монотонно убывают с ростом размера частиц.

Данные результаты согласуются с анализом индикатрис рассеяния. Увеличение диаметра частицы относительно длины волны излучения, взаимодействующего с ней, приводит к деформации индикатрисы рассеяния, как это показано на рис. 2 и 3. Диаметр частиц аэрозоля должен быть меньше или равен длине волны, а точнее, не должен превышать значение длины волны излучения, так как при увеличении диаметра частицы относительно длины волны излучения наблюдается деформация индикатрисы рассеяния в сторону обычного рассеяния, что уменьшает обратное рассеяние от частицы.

В результате можно сделать вывод, что для длины волны излучения $1.5 \mu \mathrm{m}$ оптимальный размер частицы водного аэрозоля должен быть около $0.5 \mu \mathrm{m}$. Полученные оценки показывают, что концентрация производимого аэрозоля должна достигать величин $10^{7}-10^{8} \mathrm{~m}^{-3}$. При этом в объем гауссовой перетяжки телескопа $23 \mathrm{~mm}^{3}$ должно попадать до 100 рассеивающих частиц.

Далее полученные оценки используются для моделирования и оптимизации параметров газогенераторной системы. Максимальная регистрируемая скорость истечения газово-аэрозольной смеси на выходе из микроканала определяется частотой дискретизации. Согласно формуле (1), частотное смещение излучения, 
обусловленное эффектом Доплера, для длины волны $1.5 \mu \mathrm{m} \quad(200 \mathrm{THz})$ составляет примерно $1.33 \mathrm{MHz}$ на $1 \mathrm{~m} / \mathrm{s}$ скорости газа $V_{z}$. При оцифровке сигнала с частотой $200 \mathrm{MHz}$ максимальная скорость истечения газовоаэрозольной смеси не должна превышать $75 \mathrm{~m} / \mathrm{s}$. Минимальная регистрируемая скорость истечения будет определяться уровнем фазовых шумов лазерного излучателя. Для современных высокостабильных одночастотных полупроводниковых лазеров этот показатель составляет $V_{z \min } \approx 0.2 \mathrm{~m} / \mathrm{s}$.

Удельное давление газовой системы на одно отверстие находится по формуле [13]:

$$
P=\rho V^{2} / 2 g+P_{a},
$$

где $V$ - скорость истечения аэрозоля, $P_{a}-$ нормальное атмосферное давление, $\rho$ - плотность аэрозоля. При условии, что максимальная скорость истечения аэрозоля не превосходит $100 \mathrm{~m} / \mathrm{s}$, плотность аэрозоля составляет величину порядка $4.2 \mathrm{~kg} / \mathrm{m}^{3}$, получается значение давления на одно отверстие около $1.2 \mathrm{~atm}$. Генерация водяного аэрозоля с такими параметрами возможна с помощью ультразвукового парообразователя, способного генерировать частицы воды диаметром $0.1-5 \mu \mathrm{m}$, причем водяной пар должен быть холодным для минимизации энергетических потерь, которые возникали бы вследствие образования конденсата горячим паром. Из результатов моделирования следует, что именно для полученного при оптимизации значения диаметра частиц $0.5 \mu \mathrm{m}$ давление в газовой системе должно быть таким, что с учетом количества отверстий в газотурбинной лопатке скорость истекания аэрозоля попадает в требуемый диапазон 5-100 m/s.

С другой стороны, среднюю скорость газа в микроканале можно оценить по формуле $V_{a v}=\Delta P R^{2} / 8 \mu L$, где $R$ - радиус микроканала, $\Delta P-$ разность давлений на входе и выходе из микроканала (в данном случае между давлением внутри лопатки и атмосферным), $L-$ длина канала, $\mu-$ коэффициент динамической вязкости газа при заданной температуре $\left(\mu=1.81 \cdot 10^{-5} \mathrm{~Pa} \cdot \mathrm{s}\right.$ для $\left.20^{\circ} \mathrm{C}\right)$. Данное выражение позволяет установить зависимость между регулируемыми параметрами газовоаэрозольной смеси (давление, вязкость) и основными структурными параметрами микроканала (его сечением и длиной) через среднюю скорость истечения газовоаэрозольной смеси, которая фактически и измеряется лидарным методом. Если точка фокусировки телескопа лидара будет находиться в непосредственной близости от выходного отверстия микроканала, то можно говорить о том, что измеряемая лидаром радиальная проекция скорости $V_{z}$ будет соответствовать $V_{a v}$ на выходе микроканала (с учетом геометрического фактора). При разности давлений $0.25 \mathrm{~atm}$ и комнатной температуре средняя скорость истечения газа на выходе микроканала длиной $30 \mathrm{~mm}$ и сечением $200 \mu \mathrm{m}$ составит $56-58 \mathrm{~m} / \mathrm{s}$, что будет соответствовать частотному доплеровскому сдвигу при лидарном детектировании $75-77 \mathrm{MHz}$ для длины волны $1.5 \mu \mathrm{m}$. Ширина и форма спектра сигнала после его фурье-преобразования будут эквивалентны распределению осевых проекций векторов скорости газового потока в области детектирования.

Анализ скоростей газово-аэрозольной смеси на выходе массива однотипных микроканалов позволяет с большой вероятностью обнаруживать их скрытые дефекты, закупоривания, отклонения от нормы. С учетом высокой скорости получения данных, обусловленной достаточно высокими частотами сигналов, время измерения одного микроканала может составлять не более $1 \mathrm{~s}$, это время может быть существенно сокращено при применении современных прецизионных средств 3D.

Таким образом, в работе описана и обоснована возможность эффективного неразрушающего контроля состояния охлаждающих микроканалов газотурбинных лопаток с помощью гетеродинного доплеровского лидара. Описанный метод обладает существенными преимуществами по сравнению с традиционными методами оценки качества и состояния охлаждающих микроканалов газотурбинных лопаток. Исследовано влияние диаметра аэрозольных частиц на интенсивность обратного рассеяния в целях увеличения полезного сигнала лидара. Показано, что мощность обратного рассеяния убывает с ростом диаметра частицы, причем убывание происходит с незначительными осцилляциями. На основе разработанной модели получены оценки оптимальных значений параметров газово-аэрозольной смеси, а также оценки скорости ее истечения в соответствии с выбранными критериями. Моделирование показало, что при типовых шумовых характеристиках фотоприемника в заданной полосе частот (до $100 \mathrm{MHz}$ ) и апертуре приемопередающего телескопа 50-80 mm достаточная оптическая мощность излучателя не превышает 50-100 $\mathrm{mW}$ при соотношении сигнал-шум SNR $\geqslant 50$. Полученные в рамках предложенной модели оценки применимы для оптимизации конструкционных особенностей лидара, учитывающего возможность измерения на малых расстояниях, а также для подбора доступной элементной базы его составляющих.

\section{Конфликт интересов}

Авторы заявляют, что у них нет конфликта интересов.

\section{Список литературы}

[1] Иноземцев А.А., Сандрачкий В.Л. // Газотурбинные двигатели. Пермь: ОАО „Авиадвигатель“, 2006. 1204 с.

[2] Robinson G. // Measurement of cooling holes in gas turbine blades using computed tomography.

URL: https:/www.cmsc.org/stuff/contentmgr/files/0/2bdcf766 d9d5daf6e892c46153c591d3/misc/cmsc2011_wed_gh_0800_ survice.pdf

[3] Digital optical comparator (patents pending). URL: https://www.visionxinc.com/downloads/applicationnotes/edm_drilled_holes_inspection.pdf 
[4] Половченко C.B. // Петербургский журнал электроники. 2014. № 3(80). C. 33-38.

[5] Lidar: range-resolved optical remote sensing of the atmosphere / Ed. C. Weitkamp. Berlin: Springer, 2005. 455 p.

[6] Churnside J.H., Wilson J.J., Tatarskii V.V. // Appl. Opt. 1997. V. 36. N 24. P. 6011-6020. https://doi.org/10.1364/AO.36.006011

[7] Churnside J.H., Thorne R.E. // Appl. Opt. 2005. V. 44. N 26. P. 5504-5511. https://doi.org/10.1364/AO.44.005504

[8] Лобанова М.А., Савин А.В. // Современные проблемы дистанционного зондирования Земли и космоса. 2010. Т. 7. № 4. C. 296-303.

[9] Качмарек $Ф$. Введение в физику лазеров / Пер. с польск. В.Д. Новикова; Под ред. М.Ф. Бухенского. М.: Мир, 1980. $540 \mathrm{c}$.

[10] Протопопов В.В., Устинов Н.Д. Лазерное гетеродинирование / Под ред. Н.Д. Устинова. М.: Наука, 1985. 288 с.

[11] Тимофеев Ю.М., Васильев А.А. Теоретические основы атмосферной оптики. СПб.: Наука, 2003. 474 с.

[12] Васильев А.В. // Вестн. СПбГУ. Сер. 4. Физика, химия. 1996. В. 4. № 25. C. 3-11.

[13] Александров Д.В., Зубарев А.Ю., Искакова Л.Ю. // Введение в гидродинамику. Екатеринбург: Изд-во Урал. ун-та, 2012. $112 \mathrm{c}$. 